\title{
Evaluation de la pollution physico-chimique et microbienne des eaux de puits de Dang-Ngaoundéré (Cameroun)
}

\author{
Augustin MBAWALA ${ }^{1 *}$, ABDOU $^{1}$ et Martin-Benoît NGASSOUM ${ }^{2}$ \\ ${ }^{1}$ Département de Sciences Alimentaires et Nutrition, ENSAI, Université de Ngaoundéré, B.P. 455 Ngaoundéré, \\ Cameroun. \\ ${ }^{2}$ Département Chimie Appliquée, ENSAI, Université de Ngaoundéré, B.P. 455 Ngaoundéré, Cameroun. \\ *Auteur correspondant, E-mail: mbawalaa@yahoo.fr; Tél :00 23799903785
}

\section{RESUME}

A Dang (Ngaoundéré-Cameroun), l'insuffisance de la distribution publique d'eau potable oblige la majorité de la population à recourir à l'eau de puits. La consommation d'eau impropre expose les habitants à de nombreuses maladies d'origine hydrique. Le but de ce travail est d'effectuer des caractérisations physicochimique et microbiologique des eaux de vingt puits de la localité de Dang afin d'établir d'éventuels risques sanitaires pour la population qui les consomme. La caractérisation microbiologique de ces eaux a porté sur la recherche et le dénombrement des bactéries sur des milieux de culture sélectifs par la technique de filtration sur membrane et celles des kystes et œufs de parasites selon la méthode de concentration formol-éther. Les résultats obtenus montrent que la densité des micro-organismes dépend des paramètres physico-chimiques de ces eaux de puits, notamment la teneur en fer, en matières organiques, en matières en suspension et en azote ammoniacal influence leur charge microbienne. Ces eaux hébergent de nombreux germes parmi lesquelles figurent les bactéries (Salmonella sp., Vibrio parahaemolyticus, Pseudomonas aeruginosa, coliformes fécaux, streptocoques fécaux, Clostridium sulfito-réducteurs) et les parasites (kystes d'Entamoeba histolytica et de Giardia lamblia, œufs de Cryptosporidium et d'Ascaris lombricoïdes). L'origine de la pollution fécale de ces points d'eau diffère selon les sites et peut être humaine, animale ou mixte. Ces résultats confirment les risques sanitaires à court terme, à moyen terme ou à long terme liés à la consommation d'eau et permettent d'expliquer la prévalence des maladies gastro-entériques fréquentes dans les résidences du campus universitaire de DangNgaoundéré.

() 2010 International Formulae Group. All rights reserved.

Mots clés : Eau de puits, caractérisation physico-chimique, pollution microbienne, risque sanitaire.

\section{INTRODUCTION}

Au Cameroun, l'approvisionnement en eau potable est assuré par la Société Nationale des Eaux du Cameroun (SNEC). A Ngaoundéré, moins de deux millions de $\mathrm{m}^{3}$ d'eau potable sont distribués annuellement par cette société, pour une population estimée à environ 185700 habitants (SNEC, 2004). Ce faible volume d'eau potable disponible oblige une grande partie de la population à recourir à l'eau de puits. Ainsi, à Dang (Ngaoundéré,
Cameroun), une localité abritant une Université d'Etat, l'accès à l'eau potable devient de plus en plus difficile d'autant plus que la population est sans cesse croissante. Cette croissance de la population s'accompagne de celle des activités anthropiques. Les déchets issus de l'activité humaine sont à l'origine de la pollution des eaux souterraines (Nkhuwa, 2003 ; Hassoune et al., 2006). Par ailleurs, la pollution de ces 
eaux souterraines représente un aspect très inquiétant, d'autant plus que leurs utilisations à des fins alimentaires constituent des risques sanitaires chez les consommateurs (Laferriere et al., 1996 ; El Kettani et Azzouzi, 2006). Ces risques sanitaires à moyen et à long termes sont liés le plus souvent à une mauvaise qualité physico-chimique alors que les risques à court terme peuvent résulter d'une mauvaise qualité microbiologique. La qualité microbiologique de l'eau est déterminée par la recherche et l'isolement des germes bactériens témoins de contamination fécale, considérés comme des bio-indicateurs de pollution tels que les coliformes fécaux, les streptocoques fécaux et les Clostridium sulfito-réducteurs ; les bactéries pathogènes opportunistes appartenant à certaines espèces des genres Pseudomonas, Aeromonas, Flavobacterium, Serratia et Klebsiella (OMS, 1986). Chez les sujets immunodéficients par exemple, Aeromonas hydrophila et Pseudomonas aeruginosa sont parfois responsables de diarrhées, d'infection de plaies ou d'infections génito-urinaires et oculaires (Le Minor et Veron, 1989). En plus de ces maladies endémiques qui sévissent dans les pays en voie de développement, l'Organisation Mondiale de la Santé en 2002 a recensé 578 maladies infectieuses dans 132 pays, et le choléra (causé par Vibrio cholerae) était le plus fréquent avec une diarrhée aiguë et occupait le quatrième rang. Après le choléra suivaient les fièvres typhoïde et paratyphoïdes causées par Salmonella typhi et Salmonella paratyphi respectivement. La consommation d'une eau contaminée par certains de ces germes est à l'origine des épidémies (Angulo et al., 1997 ; Balbus et Embrey, 2002).

Le développement de communautés microbiennes dans les eaux de surface, aussi bien que dans les eaux souterraines est lié aux facteurs météorologiques et à l'ensemble des caractéristiques physico-chimiques et biologiques du biotope (Gounot, 1994 ; Nola et al., 2002). Cependant, dans ces milieux, les éléments chimiques influencent fortement la survie des microorganismes présents. L'activité des bactéries nitrifiantes peut être affectée par les fortes variations des teneurs en oxygène dissous (Jayamohan et al., 1998). Les variations de température, de $\mathrm{pH}$ et des concentrations en $\mathrm{NaCl}$ du milieu peuvent affecter la croissance de Pseudomonas marginalis et son activité pectinolytique (Membre et Burlot, 1994).

L'objectif du présent travail est de déterminer quelques caractéristiques physicochimique et microbiologique des eaux de puits de Dang. Spécifiquement, il porte sur l'évaluation des principaux facteurs physicochimiques responsables de la variation de la qualité générale de ces eaux d'une part, sur la recherche dans ces eaux des germes de contamination fécale, des bactéries pathogènes-opportunistes et des parasites d'autre part. Ce travail est basé sur l'hypothèse suivante: la qualité physicochimique et microbiologique de l'eau de puits est fonction des conditions d'hygiène autour du puits.

\section{MATERIEL ET METHODES \\ Echantillonnage}

Vingt puits d'eau désignés par $\mathrm{P}_{1}, \mathrm{P}_{2}, \mathrm{P}_{3}$, $\mathrm{P}_{4}, \mathrm{P}_{5}, \mathrm{P}_{6}, \mathrm{P}_{7}, \mathrm{P}_{8}, \mathrm{P}_{9}, \mathrm{P}_{10}, \mathrm{P}_{11}, \mathrm{P}_{12}, \mathrm{P}_{13}, \mathrm{P}_{14}, \mathrm{P}_{15}, \mathrm{P}_{16}$, $\mathrm{P}_{17}, \mathrm{P}_{18}, \mathrm{P}_{19}, \mathrm{P}_{20}$, ont été choisis dans les quartiers de la localité de Dang (Figure 1), en fonction de leur aménagement, leur situation par rapport aux latrines, leur importance pour la population et des activités menées dans leurs alentours. Ils ont été échantillonnés chaque semaine pendant six mois.

$1000 \mathrm{ml}$ d'eau de puits par échantillon ont été prélevés aseptiquement dans un flacon en verre stérile, transportés au laboratoire dans une enceinte réfrigérée à $4{ }^{\circ} \mathrm{C}$ et analysés immédiatement.

\section{Analyses physico-chimiques}

Toutes les caractérisations physicochimiques des échantillons ont été réalisées suivant les techniques préconisées par Rodier (1978). Ainsi, la température de l'eau a été prise à l'aide d'un thermomètre gradué au $1 / 10$ de degré et la lecture a été faite après une immersion pendant 10 minutes. Le $\mathrm{pH}$ et la conductivité électrique ont été mesurés à l'aide d'un pH-mètre de marque PICCOLOATC et d'un résistivimètre de marque TACUSSEL CD-60, respectivement. Les matières en suspension ont été déterminées par filtration, séchage et pesée. La turbidité et les sulfates ont été déterminés par la méthode néphélométrique. Le calcium et le magnésium ont été dosés dans les échantillons par la 
méthode complexométrique. Les bicarbonates ont été déterminés à partir du dosage volumétrique de l'alcalinité. La méthode de Mohr décrite par Rodier (1978) a été utilisée pour déterminer la teneur en chlorures des échantillons. La concentration en azote ammoniacal des échantillons a été déterminée par la méthode au bleu d'indophénol décrite par Rodier (1978). La méthode à chaud décrite par Rodier (1978) a été utilisée pour déterminer les matières organiques dans les échantillons. Les teneurs en nitrates des échantillons ont été déterminées par la méthode au salicylate de sodium décrite par Rodier (1978). Les teneurs en fer dissous et en ortho-phosphates des échantillons ont été mesurées par spectrophotométrie. Ces analyses ont été faites pendant six mois, à raison d'un échantillonnage par semaine.

\section{Analyses microbiologiques}

Pour les analyses bactériologiques, une membrane en ester de cellulose (Millipore, Bedford, MA 01730) de porosité $0,45 \mu \mathrm{m}$ a été utilisée pour filtrer stérilement $100 \mathrm{ml}$ d'échantillon d'eau à analyser ou une dilution de celle-ci au moyen d'un dispositif de filtration relié à une trompe à vide (Havelaar et al., 1987 ; Ford, 1994). Le dénombrement de la flore mésophile aérobie totale, des coliformes totaux et fécaux, des streptocoques fécaux, des vibrions, des salmonelles, des Clostridium sulfito-réducteurs et de Pseudomonas aeruginosa a été effectué respectivement sur le milieu Yeast Extract Agar (YEA), le milieu à l'Eosine et au Bleu de Méthylène (EMB), le milieu Slanetz et Bartley, la gélose Thiosulfate-Citrate-BileSaccharose (TCBS), la gélose SalmonellaShigella (SS), le milieu Viande-Foie (VF) et la gélose au cétrimide (Guiraud et Galzy, 1980 ; Havelaar et al., 1987). Les milieux de culture bactériologique utilisés sont ceux de Diagnostics Pasteur (France).

La technique de concentration formoléther décrite par Cheesbrough en 1987 a été utilisée pour la recherche et le dénombrement des kystes de protozoaires, des œufs d'helminthes.

Les résultats sont exprimés en Unité Formant Colonie (UFC) par $100 \mathrm{ml}$ d'eau pour les bactéries et en nombre de kystes ou d'œufs par $\mathrm{mm}^{3}$ pour les kystes de protozoaires et les œufs d'helminthes.

\section{Analyses statistiques}

Les résultats ont été soumis à l'analyse de la variance (ANOVA) et au test de régression multiple de Duncan à l'aide du logiciel STATGRAPHICS Plus 5.0. Les valeurs moyennes des résultats des analyses physico-chimiques et microbiologiques ont été comparées et leurs différences significatives ont été déterminées au seuil de probabilité $\mathrm{p}<0,05$.

\section{RESULTATS}

\section{Paramètres physico-chimiques}

Les Tableaux $1 \mathrm{a}$ et $1 \mathrm{~b}$ indiquent les valeurs moyennes obtenues pour les principaux paramètres physico-chimiques des eaux de puits de Dang analysées.

Il ressort de ces tableaux que les températures mesurées sont comprises entre $22,0{ }^{\circ} \mathrm{C}$ et $24,0{ }^{\circ} \mathrm{C}$ avec une moyenne de 22,64 ${ }^{\circ} \mathrm{C}$. Les valeurs des températures obtenues ne dépassant pas $25{ }^{\circ} \mathrm{C}$, correspondent aux températures atmosphériques ambiantes et indiquent l'ouverture du système aquifère, donc sa vulnérabilité vis-àvis de la pollution. Ces eaux de puits sont légèrement acides avec des valeurs de $\mathrm{pH}$ comprises entre 5,09 $\pm 0,00\left(\mathrm{P}_{7}\right)$ et $6,09 \pm 0,00$ $\left(\mathrm{P}_{5}\right)$. Cela est compatible avec le milieu silicaté qui constitue la nappe aquifère. La conductivité électrique varie selon les points de prélèvement d'eau. Elle est plus élevée pour les puits $\mathrm{P}_{1}, \mathrm{P}_{2}, \mathrm{P}_{3}, \mathrm{P}_{4}$ et la valeur la plus faible est celle du puits $\mathrm{P}_{20}$. Une différence significative $(\mathrm{P}<0,05)$ entre les valeurs mesurées a été obtenue à cet effet. Ces valeurs traduiraient une très faible minéralisation et mettraient en évidence le processus d'altération dans le lessivage des éléments minéraux et la forte dilution des eaux souterraines. Les eaux de puits analysées ont des turbidités variant entre $0,64 \pm 0,01\left(\mathrm{P}_{11}\right)$ et 2,41 $\pm 0,02$ N.T.U. $\left(\mathrm{P}_{12}\right)$, avec une moyenne de $1,16 \pm 0,00$ N.T.U. La valeur moyenne de la turbidité des ces eaux est inférieure aux normes françaises et européennes qui recommandent une turbidité inférieure à 2 N.T.U. Il en découle une clarté observée pour certaines des eaux de puits de Dang qui 
s'expliquerait par leur dilution dans l'eau de pluie car les études ont été menées pendant la saison pluvieuse. La plus faible valeur des concentrations des matières en suspension dans les eaux de puits étudiées est celle du puits $\mathrm{P}_{2}(0,32 \pm 0,00 \mathrm{mg} / \mathrm{l})$, tandis que la plus grande valeur est obtenue dans le cas du puits $\mathrm{P}_{13}(3,42 \pm 0,01 \mathrm{mg} / \mathrm{l})$ avec une moyenne de $1,44 \pm 0,01 \mathrm{mg} / \mathrm{l}$. Une différence significative a été notée entre ces valeurs au seuil de probabilité $p<0,05$. Les concentrations en fer oscillent entre $0,12 \pm 0,00\left(\mathrm{P}_{13}\right)$ et $0,97 \pm 0,01$ $\mathrm{mg} / \mathrm{l}\left(\mathrm{P}_{6}\right)$. Cette concentration diffère selon les points d'échantillonnage et une différence significative $(\mathrm{P}<0,05)$ a été notée à cet effet. En moyenne, les concentrations en fer sont supérieures à 0,2 mg/l (OMS, 1986), seuil fixé pour une eau de consommation. Les teneurs en calcium et en magnésium de ces eaux de puits sont inférieures aux normes. En effet, les eaux potables peuvent contenir jusqu'à $140 \mathrm{mg} / \mathrm{l}$ de $\mathrm{Ca}$ (Rodier, 1978) et 75 - 200 $\mathrm{mg} / \mathrm{l}$ de $\mathrm{Mg}$ (OMS, 1986). La teneur la plus faible en chlorures a été enregistrée pour le puits $\mathrm{P}_{19}(5,17 \pm 0,26 \mathrm{mg} / \mathrm{l})$, alors que la plus forte a été celle du puits $\mathrm{P}_{1}(12,31 \pm 0,82 \mathrm{mg} / \mathrm{l})$ avec une moyenne de $7,74 \pm 0,80 \mathrm{mg} / \mathrm{l}$. Il n'existe pas de différences significatives ( $p>0,05)$ entre ces valeurs par puits (exceptés les puits $\mathrm{P}_{1}, \mathrm{P}_{3}$ et $\left.\mathrm{P}_{19}\right)$. La teneur en bicarbonates varie selon les points d'eau et une différence significative a été obtenue au seuil de $5 \%$. Le puits $\mathrm{P}_{4}$ a la concentration la plus élevée, soit $40,37 \pm 0,98 \mathrm{mg} / \mathrm{l}$. La plus petite concentration est de $8,13 \pm 0,41 \mathrm{mg} / \mathrm{l}$ $\left(\mathrm{P}_{13}\right)$. La teneur en bicarbonates variable selon les puits serait due à la nature des terrains traversés par les eaux de ruissellement. La teneur en matières organiques va de $0,60 \pm 0,20$ $\mathrm{mgO}_{2} / \mathrm{l}\left(\mathrm{P}_{3}\right)$ à $14,57 \pm 0,17 \mathrm{mgO}_{2} / 1\left(\mathrm{P}_{14}\right)$ avec

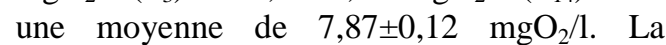
concentration moyenne en matières organiques des eaux de puits analysées est supérieure à la norme $\left(5 \mathrm{mgO}_{2} / \mathrm{l}\right)$. La teneur en sulfates, en phosphates et en nitrates des eaux de puits étudiées sont inférieures aux normes internationales (OMS, 2000). La plus faible teneur en azote ammoniacal est celle du puits $\mathrm{P}_{3}(0,09 \pm 0,01 \mathrm{mg} / \mathrm{l})$ et la plus élevée est celle $\mathrm{du}$ puits $\mathrm{P}_{8}(0,87 \pm 0,02 \mathrm{mg} / \mathrm{l})$ avec une moyenne de $0,53 \pm 0,02 \mathrm{mg} / \mathrm{l}$. Une différence significative a été notée entre les valeurs obtenues au seuil de probabilité $\mathrm{p}<0,05$.

\section{Paramètres microbiologiques}

Les résultats de l'analyse des paramètres microbiologiques des échantillons d'eau prélevés sont consignés dans le Tableau 2. Il ressort de ce tableau que la flore mésophile aérobie totale (FMAT) varie de $162,00 \cdot 10^{4} \pm 2,83 \quad \mathrm{UFC} / 100 \quad \mathrm{ml} \quad\left(\mathrm{P}_{6}\right)$ à $1344,00.10^{4} \pm 5,66 \quad \mathrm{UFC} / 100 \mathrm{ml}\left(\mathrm{P}_{14}\right)$. Les valeurs obtenues sont supérieures à celles fixées par les normes, soit $100 \mathrm{UFC} / 100 \mathrm{ml}$ (OMS, 2000) et sont significativement différentes $(p<0,05)$. En plus, la valeur de la FAMT dépasse $10^{7} \mathrm{UFC} / 100 \mathrm{ml}$ pour les puits $\mathrm{P}_{12}, \mathrm{P}_{13}, \mathrm{P}_{14}$ et $\mathrm{P}_{18}$. Le nombre de coliformes totaux fluctue entre $10,36.10^{4} \pm 1,27 \mathrm{UFC} / 100$ $\mathrm{ml}$ et $51,80.10^{4} \pm 0,27 \mathrm{UFC} / 100 \mathrm{ml}$ pour les $\mathrm{P}_{3}$ et $\mathrm{P}_{10}$, respectivement. Il en ressort que le puits $\mathrm{P}_{10}$ est 5 fois plus contaminé en coliformes totaux que le puits $\mathrm{P}_{3}$. Le dénombrement des coliformes fécaux donne une plus faible valeur pour le puits $\mathrm{P}_{15}$ $\left(4,01.10^{4} \pm 0,01 \mathrm{UFC} / 100 \mathrm{ml}\right)$, laquelle est supérieure à celle fixée par les normes, soit 20 UFC/100 ml (OMS, 2000). La concentration des streptocoques fécaux va de $12,00 \pm 1,41$ $\mathrm{UFC} / 100 \mathrm{ml}\left(\mathrm{P}_{10}\right)$ à $340,00 \pm 4,24 \mathrm{UFC} / 100 \mathrm{ml}$ $\left(\mathrm{P}_{14}\right)$. Elle est supérieure aux normes (20 UFC/100 ml), exceptés les puits $\mathrm{P}_{3}, \mathrm{P}_{6}$ et $\mathrm{P}_{10}$. En ce qui concerne Pseudomonas aeruginosa, seul le puits $\mathrm{P}_{14}$ où il $\mathrm{y}$ a absence de ce germe dans $100 \mathrm{ml}$ est conforme aux normes (OMS, 2000). La concentration en Clostridium sulfito-réducteurs de tous les puits analysés dépassent les normes (1 UFC/100 ml), exceptés les puits $\mathrm{P}_{3}$ et $\mathrm{P}_{15}$. Le nombre de vibrions dénombrés dans ces eaux de puits varie de $0\left(\mathrm{P}_{3}\right.$ et $\left.\mathrm{P}_{9}\right)$ à 287,00 $\pm 1,41 \mathrm{UFC} / 100$ $\mathrm{ml}\left(\mathrm{P}_{13}\right)$. Pour les Salmonella, les concentrations vont de $0,60.10^{4} \pm 0,14\left(\mathrm{P}_{2}\right)$ à $32,25 \cdot 10^{4} \pm 3,54 \quad \mathrm{UFC} / 100 \mathrm{ml} \quad\left(\mathrm{P}_{19}\right)$. Les concentrations en vibrions sont conformes aux normes (0 UFC/100 ml), uniquement en ce qui concerne les puits $\mathrm{P}_{3}$ et $\mathrm{P}_{9}$ tandis que celles obtenues pour les Salmonella sont toutes hors normes (absence dans $100 \mathrm{ml}$ ). Il ressort du Tableau 3 que la densité des kystes d'Entamoeba histolytica et Giardia lamblia s'échelonne respectivement entre $100,00 \pm 70,11 \quad\left(\mathrm{P}_{3}\right)$ et $2950,00 \pm 70,71$ 
kystes $/ \mathrm{mm}^{3}\left(\mathrm{P}_{1}\right)$ et entre $0\left(\mathrm{P}_{3}, \mathrm{P}_{6}, \mathrm{P}_{7}, \mathrm{P}_{9}, \mathrm{P}_{11}\right.$, $\left.\mathrm{P}_{15}, \mathrm{P}_{16}\right)$ et $275,00 \pm 35,36 \mathrm{kystes} / \mathrm{mm}^{3}\left(\mathrm{P}_{14}\right)$. Elle varie pour Cryptosporidium de $0\left(\mathrm{P}_{3}, \mathrm{P}_{6}\right.$, $\left.\mathrm{P}_{7}, \mathrm{P}_{14}, \mathrm{P}_{15}, \mathrm{P}_{18}, \mathrm{P}_{19}, \mathrm{P}_{20}\right)$ à 450,00 $\pm 141,42$ œufs $/ \mathrm{mm}^{3}\left(\mathrm{P}_{8}\right)$ et pour Ascaris lombricoüdes,

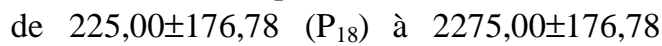

œufs $/ \mathrm{mm}^{3}\left(\mathrm{P}_{8}\right)$. Seules les eaux de puits où ont été enregistrées l'absence de kystes de Giardia lamblia et des œufs de Cryptosporidium sont conformes aux normes (OMS, 2000).

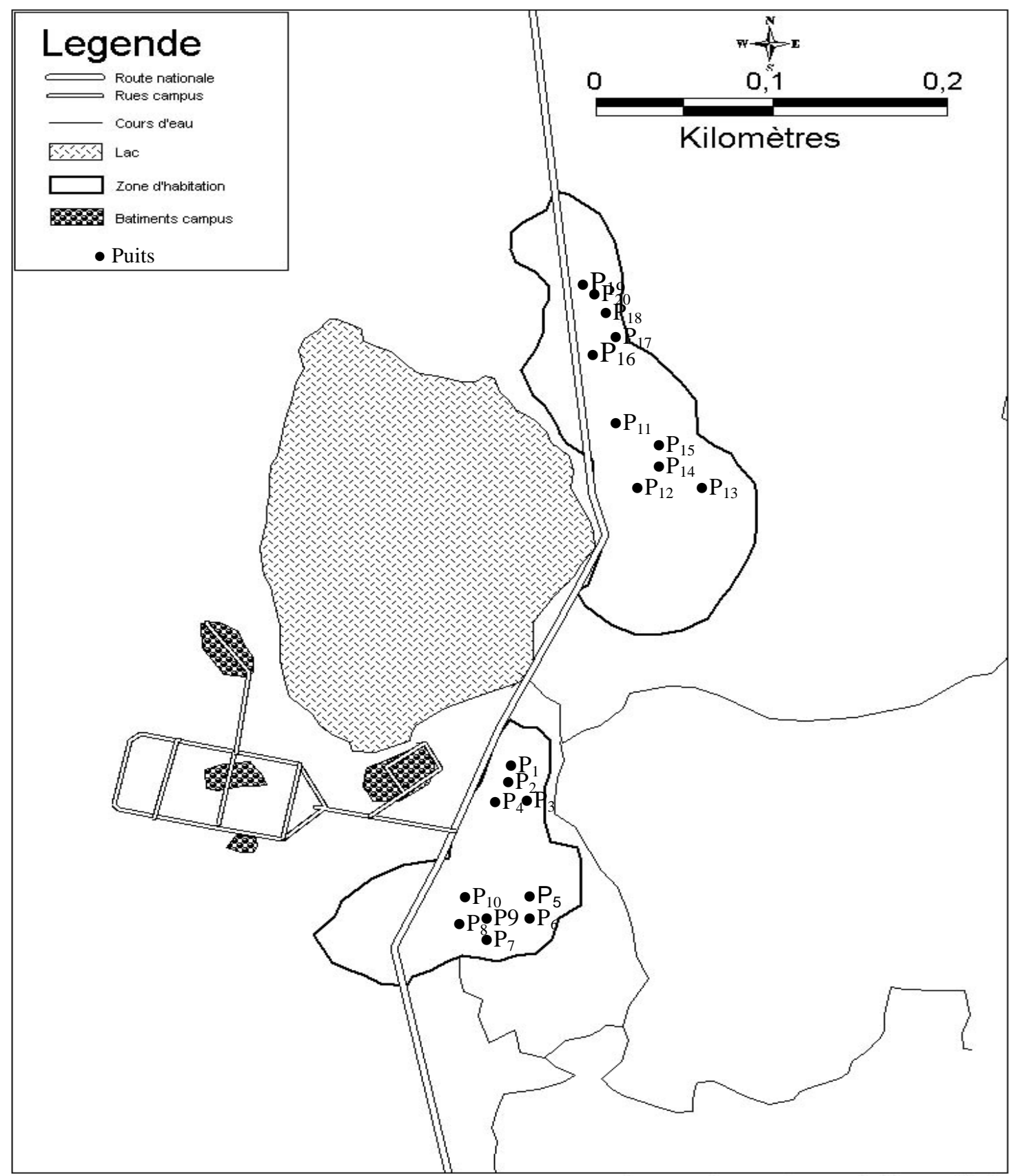

Sources: Images Raster de Ngaoundéré

Figure 1 : Localisation des points d'échantillonnage sur le site d'étude. 
A. MBAWALA et al. / Int. J. Biol. Chem. Sci. 4(6): 1962-1975, 2010

Tableau 1a : Moyennes et écarts-types des principaux paramètres physico-chimiques des eaux de puits de Dang analysées.

\begin{tabular}{|c|c|c|c|c|c|c|c|c|}
\hline Puits & $\mathbf{T}\left({ }^{\circ} \mathbf{C}\right)$ & pH & $\mathbf{X}(\boldsymbol{\mu s} / \mathbf{c m})$ & $\begin{array}{l}\text { Turbidité } \\
\text { (N.T.U.) }\end{array}$ & $\begin{array}{l}\text { MES } \\
(\mathrm{mg} / \mathrm{l})\end{array}$ & Fe (mg/l) & Ca (mg/l) & Mg (mg/l) \\
\hline $\mathrm{P}_{1}$ & $23 \pm 0^{\mathrm{h}}$ & $5,56 \pm 0^{f}$ & $51,65 \pm 0,01^{1}$ & $0,75 \pm 0^{c}$ & $0,68 \pm 0,01^{\mathrm{c}}$ & $0,17 \pm 0^{\mathrm{bc}}$ & $63,66 \pm 1,32^{i}$ & $49,34 \pm 1,17^{\mathrm{g}}$ \\
\hline $\mathrm{P}_{2}$ & $22,3 \pm 0^{c}$ & $5,47 \pm 0^{\mathrm{d}}$ & $61,13 \pm 0,02^{\circ}$ & $1,05 \pm 0^{f}$ & $0,32 \pm 0^{\mathrm{a}}$ & $0,15 \pm 0^{\mathrm{b}}$ & $123,60 \pm 3,0$ & $5,73 \pm 0,03^{\mathrm{ab}}$ \\
\hline $\mathrm{P}_{3}$ & $22,4 \pm 0^{\mathrm{d}}$ & $5,23 \pm 0^{\mathrm{b}}$ & $45,14 \pm 0,08^{1}$ & $1,5 \pm 0^{\mathrm{j}}$ & $1,42 \pm 0^{\mathrm{i}}$ & $0,13 \pm 0^{\mathrm{a}}$ & $25,39 \pm 2,32^{\mathrm{f}}$ & $6,61 \pm 1,15^{\mathrm{ab}}$ \\
\hline $\mathrm{P}_{4}$ & $22,5 \pm 0^{\mathrm{e}}$ & $5,89 \pm 0^{\mathrm{k}}$ & $51,48 \pm 0,78^{1}$ & $1,8 \pm 0^{\mathrm{k}}$ & $2,03 \pm 0,04^{\mathrm{m}}$ & $0,17 \pm 0^{\mathrm{c}}$ & $88,86 \pm 1,15^{\mathrm{j}}$ & $21,81 \pm 0,02^{\mathrm{c}}$ \\
\hline $\mathrm{P}_{5}$ & $22,4 c \pm 0^{d}$ & $6,09 \pm 0^{1}$ & $9,42 \pm 0,01^{\mathrm{f}}$ & $1,3 \pm 0^{\mathrm{h}}$ & $1,5 \pm 0,01^{\mathrm{j}}$ & $0,96 \pm 0,01$ & $17,23 \pm 1,06^{\mathrm{e}}$ & $3,77 \pm 1,32^{\mathrm{a}}$ \\
\hline $\mathrm{P}_{6}$ & $22,4 \pm 0^{\mathrm{d}}$ & $5,6 \pm 1,09^{\mathrm{g}}$ & $12,41 \pm 0,04^{\mathrm{h}}$ & $0,8 \pm 1,36^{\mathrm{d}}$ & $0,88 \pm 0,01^{\mathrm{f}}$ & $0,97 \pm 0,01$ & $17,64 \pm 3,21^{\mathrm{e}}$ & $6,70 \pm 3,41^{\mathrm{ab}}$ \\
\hline $\mathrm{P}_{7}$ & $22,5 \pm 0^{\mathrm{e}}$ & $5,09 \pm 0^{\mathrm{a}}$ & $21,43 \pm 0,13^{1}$ & $1,1 \pm 0^{\mathrm{g}}$ & $1,33 \pm 0^{\mathrm{h}}$ & $0,20 \pm 0^{\mathrm{d}}$ & $15,37 \pm 1,97^{\mathrm{e}}$ & $6,96 \pm 1,41^{\mathrm{ab}}$ \\
\hline $\mathrm{P}_{8}$ & $22,5 \pm 0^{\mathrm{e}}$ & $5,24 \pm 0^{\mathrm{b}}$ & $14,30 \pm 0,26^{\mathrm{j}}$ & $2,1 \pm 0^{\mathrm{m}}$ & $2,42 \pm 0^{\mathrm{p}}$ & $0,56 \pm 0^{\mathrm{k}}$ & $16,46 \pm 1,77^{\mathrm{e}}$ & $7,87 \pm 1,51^{\mathrm{ab}}$ \\
\hline $\mathrm{P}_{9}$ & $22,4 \pm 0^{\mathrm{d}}$ & $5,88 \pm 0^{\mathrm{k}}$ & $13,32 \pm 0,41^{\mathrm{i}}$ & $1,9 \pm 0^{1}$ & $1,59 \pm 0,01^{\mathrm{k}}$ & $0,32 \pm 0^{\mathrm{g}}$ & $15,11 \pm 1,51^{\mathrm{e}}$ & $7,89 \pm 1,51^{\mathrm{ab}}$ \\
\hline $\mathrm{P}_{10}$ & $22,6 \pm 0,1^{\mathrm{f}}$ & $5,63 \pm 0,01$ & $7 \pm 0,04^{\mathrm{b}}$ & $1,33 \pm 0^{\mathrm{i}}$ & $0,85 \pm 0,02^{\mathrm{e}}$ & $0,15 \pm 0^{\mathrm{b}}$ & $112,22 \pm 2,6$ & $11,11 \pm 3,07^{\mathrm{b}}$ \\
\hline $\mathrm{P}_{11}$ & $22,5 \pm 0^{\mathrm{e}}$ & $5,55 \pm 0,01$ & $17 \pm 0,04^{\mathrm{k}}$ & $0,64 \pm 0,01$ & $0,77 \pm 0,01^{\mathrm{d}}$ & $0,24 \pm 0^{\mathrm{f}}$ & $30,35 \pm 3,05^{\mathrm{g}}$ & $72,98 \pm 2,45^{\mathrm{j}}$ \\
\hline $\mathrm{P}_{12}$ & $22,13 \pm 0,06^{\mathrm{b}}$ & $5,80 \pm 0,01$ & $7,29 \pm 0,20^{\mathrm{bc}}$ & $2,41 \pm 0,02$ & $2,35 \pm 0,01^{\circ}$ & $0,61 \pm 0^{1}$ & $30,46 \pm 1,60^{\mathrm{h}}$ & $55,54 \pm 5,00^{\mathrm{h}}$ \\
\hline $\mathrm{P}_{13}$ & $22,3 \pm 0^{c}$ & $5,62 \pm 0^{\mathrm{h}}$ & $8,41 \pm 0,02^{\mathrm{e}}$ & $1,31 \pm 0,01$ & $3,42 \pm 0,01^{\mathrm{q}}$ & $0,12 \pm 0^{\mathrm{a}}$ & $27,25 \pm 1,61^{\mathrm{f}}$ & $63,41 \pm 8,47^{\mathrm{i}}$ \\
\hline $\mathrm{P}_{14}$ & $24 \pm 0^{\mathrm{k}}$ & $5,56 \pm 0,02$ & $9,45 \pm 0,02^{\mathrm{f}}$ & $0,85 \pm 0,01$ & $1,35 \pm 0^{\mathrm{h}}$ & $0,52 \pm 0^{i}$ & $8,02 \pm 1,61^{\mathrm{cd}}$ & $75,98 \pm 9,62^{\mathrm{j}}$ \\
\hline $\mathrm{P}_{15}$ & $22 \pm 0^{\mathrm{a}}$ & $5,52 \pm 0^{\mathrm{e}}$ & $7,85 \pm 0,03^{\mathrm{d}}$ & $0,75 \pm 0,01$ & $1,61 \pm 0,01^{1}$ & $0,32 \pm 0,01$ & $8,55 \pm 2,45^{\mathrm{cd}}$ & $95,45 \pm 2,45^{\mathrm{k}}$ \\
\hline $\mathrm{P}_{16}$ & $23,17 \pm 0,06^{\mathrm{i}}$ & $5,68 \pm 0^{\mathrm{i}}$ & $7,56 \pm 0,01^{\mathrm{cd}}$ & $0,65 \pm 0,01$ & $1,02 \pm 0,01^{\mathrm{g}}$ & $0,43 \pm 0,01$ & $6,41 \pm 1,61^{\mathrm{bc}}$ & $39,59 \pm 3,13^{\mathrm{f}}$ \\
\hline $\mathrm{P}_{17}$ & $22,77 \pm 0,06^{\mathrm{g}}$ & $5,53 \pm 0,04$ & $7,42 \pm 0,02^{\mathrm{c}}$ & $0,70 \pm 0^{\mathrm{b}}$ & $0,54 \pm 0,02^{\mathrm{b}}$ & $0,43 \pm 0^{\mathrm{h}}$ & $1,16 \pm 0,02^{\mathrm{a}}$ & $50,51 \pm 1,52^{\mathrm{g}}$ \\
\hline $\mathrm{P}_{18}$ & $23,40 \pm 0^{j}$ & $5,63 \pm 0^{\mathrm{h}}$ & $8,54 \pm 0,04^{\mathrm{e}}$ & $0,75 \pm 0^{\mathrm{c}}$ & $0,66 \pm 0,01^{\mathrm{c}}$ & $0,63 \pm 0^{1}$ & $4,15 \pm 0,99^{\mathrm{ab}}$ & $30,18 \pm 5,50^{\mathrm{d}}$ \\
\hline$P_{19}$ & $22,40 \pm 0,10^{\mathrm{d}}$ & $5,44 \pm 0^{\mathrm{c}}$ & $10,28 \pm 0,05^{\mathrm{g}}$ & $0,80 \pm 0^{\mathrm{d}}$ & $2,02 \pm 0,02^{1}$ & $0,22 \pm 0,01$ & $7,76 \pm 0,50^{\mathrm{cd}}$ & $36,24 \pm 0,50^{\mathrm{e}}$ \\
\hline $\mathrm{P}_{20}$ & $23,17 \pm 0,06^{\mathrm{i}}$ & $5,69 \pm 0,01$ & $5,60 \pm 0,04^{\mathrm{a}}$ & $0,80 \pm 0^{\mathrm{d}}$ & $2,12 \pm 0,02^{\mathrm{n}}$ & $0,54 \pm 0,04$ & $10,49 \pm 0,72^{\mathrm{d}}$ & $29,51 \pm 0,72^{\mathrm{d}}$ \\
\hline
\end{tabular}

Les valeurs n'ayant pas la même lettre en exposant sur la même colonne sont significativement différentes au seuil de probabilité $\mathrm{P}<0,05$. T $=$ température ; $\mathrm{X}=$ conductivité $; \mathrm{MES}=$ matières en suspension. 
A. MBAWALA et al. / Int. J. Biol. Chem. Sci. 4(6): 1962-1975, 2010

Tableau 1b : Moyennes et écarts-types des principaux paramètres physico-chimiques des eaux de puits de Dang analysées.

\begin{tabular}{|c|c|c|c|c|c|c|c|}
\hline Puits & $\mathrm{Cl}^{-}$(mg/l) & $\begin{array}{r}\mathrm{HCOO}_{3}^{-} \\
(\mathrm{mg} / \mathrm{l})\end{array}$ & $\begin{array}{c}\mathrm{MO} \\
\left(\mathrm{mgO}_{2} / \mathrm{l}\right)\end{array}$ & $\begin{array}{c}\mathrm{S0}_{4}^{2-} \\
(\mathrm{mg} / \mathrm{l})\end{array}$ & $\mathrm{PO}_{4}{ }^{3-}(\mathrm{mg} / \mathrm{l})$ & $\mathrm{NO}_{3}^{-}(\mathrm{mg} / \mathrm{l})$ & $\mathrm{NH}_{4}{ }^{+}(\mathrm{mg} / \mathrm{l})$ \\
\hline $\mathrm{P}_{1}$ & $12,31 \pm 0,82$ & $38,23 \pm 5,08^{i}$ & $8,72 \pm 0,12^{\mathrm{hi}}$ & $2,73 \pm 0,04^{\mathrm{f}}$ & $0,12 \pm 0,01^{\mathrm{abc}}$ & $1,44 \pm 0,08^{\mathrm{abc}}$ & $0,24 \pm 0,01^{\mathrm{b}}$ \\
\hline $\mathrm{P}_{2}$ & $8,52 \pm 0,01^{\mathrm{ef}}$ & $34,97 \pm 1,41^{\mathrm{h}}$ & $9,76 \pm 0,08^{\mathrm{k}}$ & $1,28 \pm 0,40^{\mathrm{a}}$ & $0,13 \pm 0,01^{\mathrm{abcd}}$ & $1,62 \pm 0,05^{\mathrm{abc}}$ & $0,31 \pm 0,02^{\text {cd }}$ \\
\hline $\mathrm{P}_{3}$ & $9,61 \pm 0,96^{\mathrm{g}}$ & $21,19 \pm 1,34^{\mathrm{fg}}$ & $0,60 \pm 0,20^{\mathrm{a}}$ & $2,01 \pm 0,03^{\mathrm{b}}$ & $0,17 \pm 0,11^{\text {cde }}$ & $1,42 \pm 0,01^{\mathrm{abc}}$ & $0,09 \pm 0,01^{\mathrm{a}}$ \\
\hline $\mathrm{P}_{4}$ & $6,57 \pm 0,78^{\mathrm{a}}$ & $40,37 \pm 0,98^{\mathrm{i}}$ & $12,20 \pm 0,34$ & $2,24 \pm 0^{\mathrm{c}}$ & $0,17 \pm 0,01^{\text {bcde }}$ & $1,12 \pm 0,07^{\mathrm{a}}$ & $0,29 \pm 0,02^{\mathrm{c}}$ \\
\hline $\mathrm{P}_{5}$ & $6,15 \pm 0,82^{\mathrm{a}}$ & $19,52 \pm 0^{\mathrm{ef}}$ & $9,20 \pm 0,03^{\mathrm{j}}$ & $2,21 \pm 0,06^{\mathrm{c}}$ & $0,22 \pm 0,02^{\mathrm{efg}}$ & $1,91 \pm 0,09^{\mathrm{cd}}$ & $0,52 \pm 0,04^{\mathrm{f}}$ \\
\hline $\mathrm{P}_{6}$ & $9,47 \pm 0,82^{f}$ & $21,96 \pm 0^{\mathrm{fg}}$ & $0,80 \pm 0,01^{\mathrm{a}}$ & $2,52 \pm 0,12^{\mathrm{e}}$ & $0,18 \pm 0,01^{\text {def }}$ & $1,33 \pm 0,48^{\mathrm{ab}}$ & $0,39 \pm 0,03^{\mathrm{e}}$ \\
\hline $\mathrm{P}_{7}$ & $8,52 \pm 1,42^{\mathrm{ef}}$ & $23,63 \pm 0,73^{\mathrm{g}}$ & $6,77 \pm 0,06^{\mathrm{f}}$ & $2,89 \pm 0,02^{\mathrm{f}}$ & $0,14 \pm 0,01^{\mathrm{abcd}}$ & $1,65 \pm 0,23^{b c}$ & $0,69 \pm 0,03^{\mathrm{h}}$ \\
\hline $\mathrm{P}_{8}$ & $9,47 \pm 0,82^{\mathrm{f}}$ & $21,96 \pm 0^{\mathrm{fg}}$ & $6,47 \pm 0,31^{\mathrm{e}}$ & $3,23 \pm 0,10^{\mathrm{g}}$ & $0,13 \pm 0,01^{\mathrm{abcd}}$ & $2,30 \pm 0,05^{\mathrm{de}}$ & $0,87 \pm 0,02^{\mathrm{j}}$ \\
\hline $\mathrm{P}_{9}$ & $7,75 \pm 1,95^{\mathrm{c}}$ & $19,52 \pm 2,44^{\mathrm{ef}}$ & $4,83 \pm 0,03^{\mathrm{c}}$ & $2,38 \pm 0,01^{\mathrm{c}}$ & $0,10 \pm 0,01^{\mathrm{ab}}$ & $14,56 \pm 0,07^{1}$ & $0,43 \pm 0,02^{\mathrm{e}}$ \\
\hline $\mathrm{P}_{10}$ & $6,19 \pm 0,09^{\mathrm{a}}$ & $19,77 \pm 0,26^{\mathrm{ef}}$ & $6,49 \pm 0,12^{\mathrm{e}}$ & $1,84 \pm 0,06^{b}$ & $0,09 \pm 0,01^{\mathrm{a}}$ & $3,21 \pm 0,92^{\mathrm{f}}$ & $0,35 \pm 0,02^{\mathrm{d}}$ \\
\hline $\mathrm{P}_{11}$ & $6,57 \pm 0,78^{\mathrm{a}}$ & $21,96 \pm 2,44^{\mathrm{fg}}$ & $8,80 \pm 0,04^{\mathrm{hi}}$ & $2,22 \pm 0,01^{\mathrm{c}}$ & $0,18 \pm 0^{\text {cdef }}$ & $4,18 \pm 0,09^{\mathrm{g}}$ & $0,61 \pm 0,03^{\mathrm{g}}$ \\
\hline $\mathrm{P}_{12}$ & $8,99 \pm 0,82^{\text {ef }}$ & $17,89 \pm 3,73^{\mathrm{de}}$ & $11,37 \pm 0,16$ & $2,45 \pm 0^{\mathrm{e}}$ & $0,24 \pm 0,03^{\mathrm{fg}}$ & $6,64 \pm 0,22^{\mathrm{h}}$ & $0,78 \pm 0,01^{\mathrm{i}}$ \\
\hline $\mathrm{P}_{13}$ & $5,82 \pm 0,24^{\mathrm{a}}$ & $8,13 \pm 0,41^{\mathrm{a}}$ & $11,76 \pm 0,11$ & $4,35 \pm 0,02^{\mathrm{i}}$ & $0,42 \pm 0,12^{\mathrm{hi}}$ & $12,38 \pm 0,48^{\mathrm{k}}$ & $0,82 \pm 0,02^{\mathrm{i}}$ \\
\hline $\mathrm{P}_{14}$ & $6,77 \pm 0,96^{\mathrm{b}}$ & $19,52 \pm 2,44^{\mathrm{ef}}$ & $14,57 \pm 0,17$ & $2,44 \pm 0,04^{\mathrm{d}}$ & $0,26 \pm 0,02^{\mathrm{g}}$ & $1,30 \pm 0,23^{\mathrm{ab}}$ & $0,80 \pm 0,03^{\mathrm{i}}$ \\
\hline $\mathrm{P}_{15}$ & $9,47 \pm 2,17^{\mathrm{f}}$ & $13,01 \pm 1,41^{\mathrm{c}}$ & $4,18 \pm 0,04^{b}$ & $3,60 \pm 0,04^{\mathrm{h}}$ & $0,36 \pm 0,03^{\mathrm{h}}$ & $8,58 \pm 0,51^{\mathrm{i}}$ & $0,48 \pm 0,04^{\mathrm{f}}$ \\
\hline $\mathrm{P}_{16}$ & $7,57 \pm 0,82^{\mathrm{c}}$ & $17,08 \pm 2,44^{\mathrm{de}}$ & $8,62 \pm 0,04^{\mathrm{h}}$ & $2,25 \pm 0,10^{\mathrm{c}}$ & $0,08 \pm 0,01^{\mathrm{a}}$ & $2,67 \pm 0,49^{\mathrm{e}}$ & $0,73 \pm 0,03^{\mathrm{h}}$ \\
\hline $\mathrm{P}_{17}$ & $5,53 \pm 0,55^{\mathrm{a}}$ & $15,48 \pm 0,96^{\mathrm{cd}}$ & $8,97 \pm 0,20^{\mathrm{ij}}$ & $6,32 \pm 0,27^{j}$ & $0,24 \pm 0,02^{\mathrm{fg}}$ & $11,02 \pm 0,18^{\mathrm{j}}$ & $0,81 \pm 0,03^{\mathrm{i}}$ \\
\hline $\mathrm{P}_{18}$ & $6,36 \pm 0,79^{\mathrm{a}}$ & $16,82 \pm 0,43^{\mathrm{de}}$ & $11,17 \pm 0,03$ & $4,16 \pm 0,03^{\mathrm{i}}$ & $0,43 \pm 0,03^{\mathrm{i}}$ & $1,22 \pm 0,01^{\mathrm{ab}}$ & $0,49 \pm 0,03^{\mathrm{f}}$ \\
\hline $\mathrm{P}_{19}$ & $5,17 \pm 0,26^{\mathrm{a}}$ & $12,33 \pm 0,79^{\mathrm{bc}}$ & $5,10 \pm 0,21^{\mathrm{b}}$ & $2,51 \pm 0,04^{\mathrm{e}}$ & $0,17 \pm 0^{\text {cde }}$ & $8,14 \pm 0,03^{\mathrm{i}}$ & $0,40 \pm 0,03^{\mathrm{e}}$ \\
\hline $\mathrm{P}_{20}$ & $7,90 \pm 0,09^{\mathrm{d}}$ & $9,80 \pm 0,61^{\mathrm{ab}}$ & $7,03 \pm 0,10^{\mathrm{g}}$ & $2,48 \pm 0,01^{\mathrm{e}}$ & $0,27 \pm 0,03^{\mathrm{g}}$ & $11,38 \pm 0,18^{\mathrm{j}}$ & $0,41 \pm 0,01^{\mathrm{e}}$ \\
\hline
\end{tabular}

Les valeurs n'ayant pas la même lettre en exposant sur la même colonne sont significativement différentes au seuil de probabilité $\mathrm{P}<0,05$. $\mathrm{MO}=$ matières organiques. 
A. MBAWALA et al. / Int. J. Biol. Chem. Sci. 4(6): 1962-1975, 2010

Tableau 2 : Moyennes et écarts-types des principaux germes bactériens dénombrés dans les eaux de puits de Dang analysées.

\begin{tabular}{|c|c|c|c|c|c|c|c|c|}
\hline Puits & $\begin{array}{c}\text { FMAT } \\
\text { (UFC/100ml) }\end{array}$ & $\begin{array}{c}\text { CT } \\
(\mathrm{UFC} / 100 \mathrm{ml})\end{array}$ & $\begin{array}{c}\text { CF } \\
(\mathrm{UFC} / 100 \mathrm{ml})\end{array}$ & $\begin{array}{c}\text { SF } \\
(\mathrm{UFC} / 100 \mathrm{ml})\end{array}$ & $\begin{array}{c}\text { Pa } \\
(\mathrm{UFC} / 100 \mathrm{ml})\end{array}$ & $\begin{array}{c}\mathrm{Cl} \\
(\mathrm{UFC} / 100 \mathrm{ml})\end{array}$ & $\begin{array}{c}\mathrm{V} \\
(\mathrm{UFC} / \mathbf{1 0 0 m l})\end{array}$ & $\begin{array}{c}\mathrm{S} \\
(\mathrm{UFC} / \mathbf{1 0 0 m l})\end{array}$ \\
\hline $\mathrm{P}_{1}$ & $664.10^{4} \pm 5,66^{\mathrm{f}}$ & $30,8 \cdot 10^{4} \pm 0,28^{\mathrm{ghi}}$ & $17,2.10^{4} \pm 0,28^{j}$ & $101 \pm 1,41^{\mathrm{d}}$ & $60 \pm 4,24^{\mathrm{f}}$ & $80 \pm 0^{f}$ & $80 \pm 1,41^{\mathrm{h}}$ & $24,55.10^{4} \pm 0,071^{\mathrm{h}}$ \\
\hline $\mathrm{P}_{2}$ & $874.10^{4} \pm 8,49^{\mathrm{i}}$ & $35,5 \cdot 10^{4} \pm 0,42^{i j}$ & $20,3 \cdot 10^{4} \pm 0,71^{\mathrm{k}}$ & $95,5 \pm 2,12^{\mathrm{d}}$ & $132 \pm 2,83^{\mathrm{i}}$ & $40 \pm 0^{\mathrm{cd}}$ & $88 \pm 2,83^{\mathrm{i}}$ & $0,6 \cdot 10^{4} \pm 0,14^{\mathrm{a}}$ \\
\hline $\mathrm{P}_{3}$ & $178.10^{4} \pm 19,80^{\mathrm{a}}$ & $10,36 \cdot 10^{4} \pm 1,27^{\mathrm{a}}$ & $9,1 \cdot 10^{4} \pm 0,14^{\mathrm{cd}}$ & $16 \pm 1,41^{\mathrm{a}}$ & $43,5 \pm 3,54^{\mathrm{e}}$ & $0^{\mathrm{a}}$ & $0^{\mathrm{a}}$ & $8,8 \cdot 10^{4} \pm 0,14^{\mathrm{d}}$ \\
\hline $\mathrm{P}_{4}$ & $276.10^{4} \pm 28,28^{\mathrm{b}}$ & $42,6.10^{4} \pm 0,56^{\mathrm{kl}}$ & $26,5 \cdot 10^{4} \pm 0,42^{\mathrm{m}}$ & $210,5 \pm 2,12^{\mathrm{h}}$ & $101,5 \pm 3,54^{\mathrm{h}}$ & $20 \pm 0^{\mathrm{b}}$ & $22 \pm 1,42^{\mathrm{d}}$ & $29,6.10^{4} \pm 0,14^{\mathrm{kl}}$ \\
\hline $\mathrm{P}_{5}$ & $462 \cdot 10^{4} \pm 25,46^{\mathrm{d}}$ & $49,7 \cdot 10^{4} \pm 0,71^{\mathrm{mn}}$ & $22.10^{4} \pm 0,85^{1}$ & $34 \pm 1,41^{\mathrm{b}}$ & $25,5 \pm 0,71^{\mathrm{c}}$ & $40 \pm 0^{\text {cd }}$ & $55 \pm 2,83^{\mathrm{f}}$ & $25,9 \cdot 10^{4} \pm 0,57^{\mathrm{hi}}$ \\
\hline $\mathrm{P}_{6}$ & $162.10^{4} \pm 2,83^{\mathrm{a}}$ & $13,7 \cdot 10^{4} \pm 0,14^{\mathrm{ab}}$ & $6,3 \cdot 10^{4} \pm 0,14^{\mathrm{b}}$ & $20 \pm 1,41^{\mathrm{a}}$ & $27 \pm 4,24^{\mathrm{c}}$ & $20 \pm 0^{\mathrm{b}}$ & $65 \pm 1,41^{\mathrm{g}}$ & $3,95 \cdot 10^{4} \pm 0,21^{\mathrm{b}}$ \\
\hline $\mathrm{P}_{7}$ & $882.10^{4} \pm 0,8^{\mathrm{i}}$ & $29,8.10^{4} \pm 0,57^{\text {gghi }}$ & $8,8 \cdot 10^{4} \pm 0,28^{\mathrm{cd}}$ & $82 \pm 1,41^{\mathrm{c}}$ & $23 \pm 5,66^{\mathrm{bc}}$ & $40 \pm 0^{\mathrm{cd}}$ & $2 \pm 0^{\mathrm{a}}$ & $13,45 \cdot 10^{4} \pm 0,21^{\mathrm{e}}$ \\
\hline $\mathrm{P}_{8}$ & $950.10^{4} \pm 0,8^{\mathrm{j}}$ & $45,6 \cdot 10^{4} \pm 0,85^{\mathrm{lm}}$ & $11,6.10^{4} \pm 0,28^{f}$ & $159 \pm 5,66^{\mathrm{g}}$ & $127,5 \pm 4,95^{\mathrm{i}}$ & $60 \pm 0^{\mathrm{e}}$ & $98 \pm 1,41^{\mathrm{g}}$ & $28,25 \cdot 10^{4} \pm 0,78^{\mathrm{jk}}$ \\
\hline $\mathrm{P}_{9}$ & $456.10^{4} \pm 5,66^{\mathrm{d}}$ & $48,1.10^{4} \pm 1,56^{\mathrm{lmn}}$ & $38 \cdot 10^{4} \pm 0,85^{\mathrm{n}}$ & $250 \pm 21,21^{\mathrm{j}}$ & $19 \pm 2,83^{\mathrm{b}}$ & $60 \pm 0^{\mathrm{e}}$ & $0^{\mathrm{a}}$ & $17,05 \cdot 10^{4} \pm 3,75^{\mathrm{f}}$ \\
\hline $\mathrm{P}_{10}$ & $516.10^{4} \pm 11,31^{\mathrm{e}}$ & $51,8 \cdot 10^{4} \pm 2,27^{\mathrm{n}}$ & $16,95 \cdot 10^{4} \pm 0,07^{\mathrm{j}}$ & $12 \pm 1,41^{\mathrm{a}}$ & $58,5 \pm 3,54^{\mathrm{f}}$ & $80 \pm 0^{\mathrm{f}}$ & $2 \pm 0^{\mathrm{a}}$ & $27,2 \cdot 10^{4} \pm 0,42^{\mathrm{ij}}$ \\
\hline$P_{11}$ & $882.10^{4} \pm 14,14^{\mathrm{i}}$ & $11.10^{4} \pm 0,56^{\mathrm{a}}$ & $6,5 \cdot 10^{4} \pm 0,14^{\mathrm{b}}$ & $219 \pm 2,83^{\text {hi }}$ & $184 \pm 1,42^{\mathrm{j}}$ & $20 \pm 0^{\mathrm{b}}$ & $134 \pm 1,42^{\mathrm{k}}$ & $6,8 \cdot 100^{4} \pm 0,28^{c}$ \\
\hline $\mathrm{P}_{12}$ & $1152.10^{4} \pm 0^{1}$ & $27,4 \cdot 10^{4} \pm 0,28^{\mathrm{fgh}}$ & $12,7 \cdot 10^{4} \pm 0,14^{\mathrm{g}}$ & $284,5 \pm 4,95^{\mathrm{k}}$ & $286,5 \pm 0,71^{1}$ & $60 \pm 0^{\mathrm{e}}$ & $260,5 \pm 7,78^{\mathrm{m}}$ & $21,4 \cdot 10^{4} \pm 1,41^{\mathrm{g}}$ \\
\hline $\mathrm{P}_{13}$ & $1294.10^{4} \pm 19,80^{\mathrm{m}}$ & $24,2 \cdot 10^{4} \pm 1,41^{\mathrm{def}}$ & $11,9 \cdot 10^{4} \pm 0,14^{\mathrm{f}}$ & $326,5 \pm 9,19^{1}$ & $84 \pm 1,41^{\mathrm{g}}$ & $50 \pm 14,14^{\mathrm{de}}$ & $287 \pm 1,41^{\mathrm{n}}$ & $30,9 \cdot 10^{4} \pm 0,28^{\mathrm{Im}}$ \\
\hline $\mathrm{P}_{14}$ & $1344.10^{4} \pm 5,66^{\mathrm{n}}$ & $38,6 \cdot 10^{4} \pm 0,28^{\mathrm{jk}}$ & $17,3 \cdot 10^{4} \pm 0,14^{\mathrm{j}}$ & $340 \pm 4,24^{\mathrm{m}}$ & $0^{\mathrm{a}}$ & $20 \pm 0^{\mathrm{b}}$ & $236 \pm 1,41^{1}$ & $27,2 \cdot 100^{4} \pm 0,28^{\mathrm{ij}}$ \\
\hline $\mathrm{P}_{15}$ & $310.10^{4} \pm 2,83^{c}$ & $15,35 \cdot 10^{4} \pm 0,71^{\mathrm{abc}}$ & $4,01 \cdot 10^{4} \pm 0,01^{\mathrm{a}}$ & $81,5 \pm 6,36^{\mathrm{c}}$ & $267,5 \pm 2,12^{\mathrm{k}}$ & $0^{\mathrm{a}}$ & $21 \pm 1,41^{\mathrm{d}}$ & $14,15 \cdot 10^{4} \pm 0,07^{\mathrm{e}}$ \\
\hline $\mathrm{P}_{16}$ & $946.10^{4} \pm 8,49^{\mathrm{j}}$ & $31,95 \cdot 10^{4} \pm 707,11^{\mathrm{hi}}$ & $15,45 \cdot 10^{4} \pm 0,07^{\mathrm{i}}$ & $144 \pm 2,83^{\mathrm{f}}$ & $1 \pm 0^{\mathrm{a}}$ & $30 \pm 14,14^{\mathrm{bc}}$ & $48 \pm 1,41^{\mathrm{e}}$ & $26,65 \cdot 10^{4} \pm 0,07^{\mathrm{ij}}$ \\
\hline $\mathrm{P}_{17}$ & $946.10^{4} \pm 8,49^{\mathrm{j}}$ & $25,55 \cdot 10^{4} \pm 0,07^{\mathrm{efg}}$ & $10,75 \cdot 10^{4} \pm 0,07^{\mathrm{e}}$ & $167,5 \pm 7,78^{\mathrm{g}}$ & $37 \pm 1,41^{\mathrm{d}}$ & $30 \pm 14,14^{\mathrm{bc}}$ & $48,5 \pm 2,12^{\mathrm{e}}$ & $13,3 \cdot 10^{4} \pm 0,42^{\mathrm{e}}$ \\
\hline $\mathrm{P}_{18}$ & $1068.10^{4} \pm 16,97^{\mathrm{k}}$ & $20,95 \cdot 10^{4} \pm 0,21^{\text {cde }}$ & $9,55 \cdot 10^{4} \pm 0,07^{\mathrm{d}}$ & $146 \pm 1,41^{\mathrm{f}}$ & $1 \pm 0^{\mathrm{a}}$ & $60 \pm 0^{\mathrm{e}}$ & $13 \pm 2,83^{\mathrm{c}}$ & $25,7.10^{4} \pm 1,41^{\mathrm{hi}}$ \\
\hline $\mathrm{P}_{19}$ & $747,25 \cdot 10^{4} \pm 1,06^{\mathrm{g}}$ & $18,9 \cdot 10^{4} \pm 0,14^{\text {bcd }}$ & $8,4 \cdot 10^{4} \pm 0,28^{c}$ & $231 \pm 4,24^{\mathrm{i}}$ & $46 \pm 2,83^{\mathrm{e}}$ & $80 \pm 0^{f}$ & $19 \pm 1,41^{\mathrm{d}}$ & $32,25 \cdot 10^{4} \pm 3,54^{\mathrm{m}}$ \\
\hline $\mathrm{P}_{20}$ & $799.10^{4} \pm 1,414^{\mathrm{h}}$ & $34,9 \cdot 10^{4} \pm 1,27^{\mathrm{ij}}$ & $14,15 \cdot 10^{4} \pm 0,35^{\mathrm{h}}$ & $119 \pm 2,83^{\mathrm{e}}$ & $4 \pm 0^{\mathrm{a}}$ & $40 \pm 0^{\mathrm{cd}}$ & $7 \pm 1,41^{\mathrm{b}}$ & $16,55 \cdot 10^{4} \pm 0,07^{\mathrm{f}}$ \\
\hline
\end{tabular}

colonie $; \mathrm{CT}=$ coliformes totaux $; \mathrm{CF}=$ coliformes fécaux $; \mathrm{SF}=$ streptocoques fécaux $; \mathrm{Pa}=$ Pseudomonas aeruginosa $; \mathrm{Cl}=$ Clostridium sulfito-réducteurs $; \mathrm{V}=$ vibrions $; \mathrm{S}=$ Salmonella sp. 
A. MBAWALA et al. / Int. J. Biol. Chem. Sci. 4(6): 1962-1975, 2010

Tableau 3 : Kystes de protozoaires et œufs d'helminthes isolés des eaux de puits de Dang analysées (en unité/mm³).

\begin{tabular}{|c|c|c|c|c|}
\hline Puits & E. histolytica & G. lamblia & Cryptosporidium & A. lombricoüdes \\
\hline $\mathrm{P}_{1}$ & $2950 \pm 70,71^{\mathrm{i}}$ & $200 \pm 70,71^{\mathrm{ef}}$ & $175 \pm 35,36^{\text {bcde }}$ & $975 \pm 106,07^{\mathrm{bcd}}$ \\
\hline $\mathrm{P}_{2}$ & $2175 \pm 176,71^{\mathrm{h}}$ & $50 \pm 0,00^{\mathrm{ab}}$ & $325 \pm 35,36^{\mathrm{fg}}$ & $925 \pm 106,07^{\mathrm{bcd}}$ \\
\hline $\mathrm{P}_{3}$ & $100 \pm 70,11^{\mathrm{a}}$ & $0^{\mathrm{a}}$ & $0^{\mathrm{a}}$ & $250 \pm 70,71^{\mathrm{a}}$ \\
\hline $\mathrm{P}_{4}$ & $2500 \pm 848,53^{\mathrm{h}}$ & $100 \pm 70,71^{\mathrm{bcd}}$ & $50 \pm 0,00^{\mathrm{ab}}$ & $975 \pm 247,49^{\mathrm{bcd}}$ \\
\hline $\mathrm{P}_{5}$ & $475 \pm 106,07^{\text {abcd }}$ & $50 \pm 70,71^{\mathrm{ab}}$ & $275 \pm 106,07^{\mathrm{ef}}$ & $1150 \pm 212,13^{\text {cde }}$ \\
\hline $\mathrm{P}_{6}$ & $925 \pm 06,07^{\text {ef }}$ & $0^{\mathrm{a}}$ & $0^{\mathrm{a}}$ & $950 \pm 141,42^{\mathrm{bcd}}$ \\
\hline $\mathrm{P}_{7}$ & $1700 \pm 70,71^{\mathrm{g}}$ & $0^{\mathrm{a}}$ & $0^{\mathrm{a}}$ & $925 \pm 176,78^{\mathrm{bcd}}$ \\
\hline $\mathrm{P}_{8}$ & $2250 \pm 141,42^{\mathrm{h}}$ & $125 \pm 35,36^{\text {bcde }}$ & $450 \pm 141,42^{\mathrm{g}}$ & $2275 \pm 176,78^{\mathrm{g}}$ \\
\hline $\mathrm{P}_{9}$ & $200 \pm 0,00^{\mathrm{ab}}$ & $0^{\mathrm{a}}$ & $100 \pm 0,00^{\mathrm{abc}}$ & $575 \pm 176,78^{\mathrm{ab}}$ \\
\hline $\mathrm{P}_{10}$ & $275 \pm 35,36^{\mathrm{abc}}$ & $100 \pm 0,00^{\mathrm{bcd}}$ & $200 \pm 141,42^{\text {cdef }}$ & $1100 \pm 353,55^{\text {cde }}$ \\
\hline $\mathrm{P}_{11}$ & $700 \pm 70,71^{\text {cdef }}$ & $0^{\mathrm{a}}$ & $175 \pm 35,36^{\text {bcde }}$ & $1525 \pm 176,78^{\text {ef }}$ \\
\hline $\mathrm{P}_{12}$ & $775 \pm 106,07^{\text {def }}$ & $200 \pm 70,71^{\mathrm{ef}}$ & $200 \pm 0,00^{\text {cdef }}$ & $1800 \pm 212,13^{\mathrm{f}}$ \\
\hline $\mathrm{P}_{13}$ & $1575 \pm 176,78^{\mathrm{g}}$ & $175 \pm 106,07^{\mathrm{de}}$ & $250 \pm 141,42^{\text {def }}$ & $1000 \pm 212,13^{\mathrm{bcd}}$ \\
\hline $\mathrm{P}_{14}$ & $775 \pm 35,36^{\mathrm{def}}$ & $275 \pm 35,36^{\mathrm{f}}$ & $0^{\mathrm{a}}$ & $1000 \pm 282,84^{\mathrm{bcd}}$ \\
\hline $\mathrm{P}_{15}$ & $400 \pm 0,00^{\mathrm{abcd}}$ & $0^{\mathrm{a}}$ & $0^{\mathrm{a}}$ & $825 \pm 106,07^{\mathrm{bc}}$ \\
\hline $\mathrm{P}_{16}$ & $1100 \pm 70,71^{\mathrm{f}}$ & $0^{\mathrm{a}}$ & $125 \pm 35,36^{\mathrm{abcd}}$ & $625 \pm 176,78^{\mathrm{ab}}$ \\
\hline $\mathrm{P}_{17}$ & $975 \pm 35,36^{\mathrm{ef}}$ & $175 \pm 35,36^{\mathrm{ef}}$ & $50 \pm 0,00^{\mathrm{ab}}$ & $775 \pm 176,78^{\mathrm{bc}}$ \\
\hline $\mathrm{P}_{18}$ & $400 \pm 70,71 \mathrm{abcd}$ & $75 \pm 35,36^{\mathrm{abc}}$ & $0^{\mathrm{a}}$ & $225 \pm 176,78^{a}$ \\
\hline $\mathrm{P}_{19}$ & $600 \pm 70,71^{\text {bcde }}$ & $50 \pm 0,00^{\mathrm{ab}}$ & $0^{\mathrm{a}}$ & $1150 \pm 353,55^{\text {cde }}$ \\
\hline $\mathrm{P}_{20}$ & $350 \pm 70,71^{\mathrm{abcd}}$ & $150 \pm 0,00^{\text {cde }}$ & $0^{\mathrm{a}}$ & $1375 \pm 388,91^{\mathrm{def}}$ \\
\hline
\end{tabular}

Les valeurs n'ayant pas la même lettre en exposant sur la même colonne sont significativement différentes au seuil de probabilité $\mathrm{P}<0,05$ 


\section{DISCUSSION}

Les valeurs moyennes de $\mathrm{pH}$ mesurées inférieures à 7,0 justifient le fait selon lequel les propriétés chimiques d'une eau souterraine ne sont pas significativement différentes de celles du sol qui l'encaisse et la protège (Faillat, 1990). Cependant, le $\mathrm{pH}$ varie suivant les puits et une différence significative a été obtenue $(\mathrm{P}<0,05)$ traduisant l'influence de diverses sources de pollution proches des puits. En effet, la qualité physico-chimique des eaux souterraines peu profondes dépend bien souvent de l'activité humaine, tant agricole que domestique, (Close et al., 1989; Hassoune et al., 2006), du type de végétation recouvrant le sol (Mahendrappa, 1989).

Les concentrations en matières en suspension des eaux sont semblables à ceux de Lewa (2005). Elles traduisent une pollution par les argiles, les limons, les débris de la litière, etc., qui serait due au ruissellement des eaux pluviales qui charrient les débris de toutes sortes et les déposent dans les eaux de puits lorsque ceux-ci ne sont pas protégés par un couvercle et une margelle haute. Ceci n'est pas sans conséquences malgré leur faible concentration, car les matières en suspension peuvent constituer un vecteur pour l'absorption des substances toxiques par l'organisme. Selon la norme de l'OMS (1986), elles doivent être absentes des eaux de boisson, et pour les eaux de vaisselle et de lessive, inférieures à $0,05 \mathrm{mg} / \mathrm{l}$.

L'importance du fer dans les eaux supérieures aux normes serait liée à la forte érosion des sols (par ailleurs de nature latéritique, ferrugineux) de la zone d'étude constatée par Tchotsoua et al. (1998) d'une part, et d'autre part à la pollution d'origine anthropique notamment les déchets domestiques et les eaux usées. Cette pollution s'accentue avec la croissance démographique (Tchotsoua et al., 1999).

Les chlorures présents dans les eaux de puits de Dang ont pour origine les eaux usées et déchets domestiques d'une part, et la nature du sol d'autre part. En effet, la nature des terrains traversés par les eaux souterraines influence significativement la teneur en chlorures de ces dernières (Rodier, 1978; Faillat, 1990 ; Tchotsoua et al., 1998).

L'importance des matières organiques présentes dans les eaux de puits à des concentrations supérieures à la norme $5 \mathrm{mg}$ $\mathrm{O}_{2} / \mathrm{ml}$ (exceptés les puits $\mathrm{P}_{3}, \mathrm{P}_{6}, \mathrm{P}_{9}$ et $\mathrm{P}_{15}$ ) dénonce une influence importante de l'activité de l'homme autour de ces points d'eau. Cette pollution trouve très probablement ses origines dans l'insuffisance des infrastructures d'assainissement et de collecte des ordures ménagères. En effet, dans ce site d'étude, la population ne dispose pas d'un système de collecte, d'évacuation et de traitement des ordures ménagères, encore moins de celui de collecte, de traitement, d'évacuation des eaux usées. Généralement, les ordures sont jetées pêle-mêle dans le village et les eaux usées sont directement déversées au sol dans la cour ou dans les rues.

La présence de phosphates dans ces eaux est plus importante que celle des eaux de quelques sources (lahorés) de Ngaoundéré et de Tignère (0,01-0,04 mg/l) (Fezeu, 2000). Ceci pourrait être dû à l'effet de la pollution d'origine humaine (déchets domestiques, eaux usées), à la nature des terrains traversés, au lessivage des sols cultivés où les engrais sont employés pour la fertilisation (cas le plus fréquent) et à l'utilisation des détergents pour le lavage des vêtements.

Les nitrates sont présents dans ces eaux de puits à des concentrations inférieures à la norme car les eaux de consommation peuvent en contenir jusqu'à 50 mg/l (Cardot, 2002). Cependant, ces concentrations peuvent favoriser le développement des bactéries nitrifiantes. L'origine de ces nitrates serait les engrais, les déjections humaines et animales.

$\mathrm{La}$ teneur moyenne en azote ammoniacal des eaux analysées est non conforme à la norme européenne qui préconise comme valeur maximale $0,05 \mathrm{mg} / \mathrm{l}$ pour l'eau de boisson. Cette pollution est inversement proportionnelle à la profondeur des puits. Ce qui signifie que lorsqu'un puits est profond, il est moins riche en azote ammoniacal. D'ailleurs, nous avons déterminé une corrélation négative et significative au 
seuil de $5 \%(r=0,60 ; p=0,01)$ en ce qui concerne ces paramètres. La présence de l'azote ammoniacal serait liée à la pollution d'origine humaine (pollution par les déchets domestiques et les eaux usées domestiques) en plus de la dégradation partielle des matières organiques contenues dans l'eau.

La charge microbienne importante dans les eaux de puits analysées pourrait se justifier par un certain nombre de facteurs inhérents aux activités agro-pastorales autour des puits, un défaut d'aménagement de ces puits, la proximité des sources de pollution telles que les ordures ménagères, les latrines et le non respect des règles d'hygiène élémentaire par les utilisateurs. Les plus grandes valeurs de la FMAT notées au niveau des puits $\mathrm{P}_{12}, \mathrm{P}_{13}, \mathrm{P}_{14}$ et $\mathrm{P}_{18}$ et qui sont toutes supérieures à $10^{7}$ UFC/100 ml d'eau confirment l'assertion selon laquelle la profondeur des puits et la distance entre ceux-ci et les latrines influencent la prolifération des germes dans l'eau des puits (Aissi, 1992 ; Amegee, 1992). La distance entre ces puits et les latrines est de $5,8,13$ et $27 \mathrm{~m}$ pour les $\mathrm{P}_{14}, \mathrm{P}_{12}, \mathrm{P}_{13}$ et $\mathrm{P}_{18}$ respectivement. Une corrélation négative $(r=$ - 0,44), mais significative au seuil de $5 \%$ a été trouvée, ce qui veut dire ici que lorsque la distance entre les puits et les latrines diminue, la concentration en FMAT augmente. La profondeur des puits ci-dessus cités est de 8 , 8,9 et $11 \mathrm{~m}$ pour les $\mathrm{P}_{13}, \mathrm{P}_{12}, \mathrm{P}_{18}$ et $\mathrm{P}_{14}$ respectivement. Nous avons déterminé également une corrélation négative $(\mathrm{r}=$ - 0,60), mais significative au seuil de 5\%. Ceci démontre que moins le puits est profond, plus il est pollué.

Par ailleurs, une corrélation positive $(r=$ 0,71 ) et significative au seuil de $5 \%$ entre la FMAT et la matière organique d'une part et une corrélation positive $(\mathrm{r}=0,75)$ et significative au seuil de 5\% entre la FMAT et l'azote ammoniacal $\left(\mathrm{NH}_{4}\right)$ d'autre part, ont été trouvées. Ces résultats sont comparables à ceux trouvés par Nola et al. (2002) dans les eaux souterraines de la région du Centre (Cameroun).

Les variations des résultats de dénombrement des coliformes totaux et des coliformes fécaux, seraient dues à la distance entre les puits et les latrines. La distance entre le puits le moins contaminé $\left(\mathrm{P}_{3}\right)$ et l'installation septique la plus proche est de 25 $\mathrm{m}$ tandis que celle entre le puits le plus pollué $\left(\mathrm{P}_{10}\right)$ est de $3 \mathrm{~m}$. Une corrélation négative $(\mathrm{r}=$ - 0,48$)$ et significative au seuil de $5 \%$ a été trouvée pour ces paramètres. Il est remarquable que moins les toilettes sont éloignés des puits, plus la population de coliformes totaux est importante. Le nombre de coliformes fécaux croît avec le $\mathrm{pH}$ des eaux. Une corrélation positive $(\mathrm{r}=0,58)$ au seuil de 5\% a été trouvée. Ces bactéries coliformes sont rencontrées largement dans les fèces d'origine animale et humaine (Kabler et Clark, 1961). C'est pourquoi elles se retrouvent en abondance dans les puits situés près des installations septiques. Ce phénomène est beaucoup plus accentué lorsque les fosses septiques se retrouvent en amont ou au même niveau que les puits. Tels sont les cas des $\mathrm{P}_{2}, \mathrm{P}_{3}, \mathrm{P}_{4}, \mathrm{P}_{16}, \mathrm{P}_{17}, \mathrm{P}_{18}, \mathrm{P}_{19}$ et $\mathrm{P}_{20}$. Les microbes, se déplaçant dans le sens de l'écoulement de l'eau, atteindront facilement et rapidement les eaux de puits lorsque que ces dernières sont proches des sources de pollution. Dans le même sens, Mintz et al. (1995) ont remarqué une contamination des eaux souterraines par infiltration des matières fécales.

La mise en évidence des streptocoques fécaux dans l'eau témoigne d'une pollution fécale récente de l'eau de puits (Clausen et al., 1977). En effet, les streptocoques fécaux sont caractéristiques des déjections animale et humaine (Farrow, 1984; Bitton, 1999). La présence de ces germes dans l'eau de puits de Dang serait due à la contamination par les fèces des animaux (bœufs, moutons, chèvres et volailles) en divagation aux alentours des puits. En plus, le puisoir traîne sur le sol et lors du puisage, il est introduit le plus souvent sans nettoyage dans le puits, y introduisant ainsi de nombreux micro-organismes. Lors des grandes averses de pluie, des quantités importantes de débris organiques sont rejetés dans les puits par le vent lorsque ceux-ci sont ouverts. Les eaux de ruissellement, à cause 
des propriétés physiques du sol, pourraient favoriser la pollution de la nappe phréatique en entraînant dans le sol et jusqu'aux eaux souterraines, de nombreuses cellules et particules. Certaines de ces particules peuvent contenir des substances nutritives pouvant induire une hausse du taux de croissance des bactéries présentes. D'ailleurs, nous avons trouvé une corrélation positive et significative $(\mathrm{r}=0,63, \mathrm{P}=0,001 ; \mathrm{r}=0,55, \mathrm{P}=0,05$ et $\mathrm{r}=$ $0,49, \mathrm{P}=0,05)$ entre les streptocoques fécaux et la matière organique, l'azote ammoniacal et la matière en suspension, respectivement. Ces résultats sont en accord avec ceux de Hassoune et al. (2010) qui ont analysé les eaux souterraines de la ville de Settat au Maroc et ont trouvé une contamination en coliformes et en streptocoques fécaux dépassant les valeurs indiquées par les normes internationales (OMS, 2000). Ces auteurs ont incriminé les eaux usées comme origine de cette contamination.

La densité des vibrions dans les eaux analysées est négativement corrélée à la profondeur des puits $(\mathrm{r}=-0,54 ; \mathrm{P}=0,01)$. Ainsi, les $\mathrm{P}_{3}$ et $\mathrm{P}_{9}$ dont les profondeurs sont de $13 \mathrm{~m}$ et $15 \mathrm{~m}$ respectivement, ne contiennent pas de vibrions, tandis le $\mathrm{P}_{13}$ profond de $8 \mathrm{~m}$ possède la plus grande densité de vibrions. Ceci s'expliquerait par le fait que les eaux polluées s'infiltrent et atteignent rapidement l'eau des puits qui ne sont pas profonds avec une charge importante de matière organique refermant des substances nutritives qui vont favoriser la croissance des bactéries. A cet effet, une corrélation positive et significative au seuil de $5 \%$ entre la densité moyenne de vibrions et la matière organique $(\mathrm{r}=0,58)$ et l'azote ammoniacal $(\mathrm{r}=0,59)$ a été trouvée. Concernant les salmonelles, le $\mathrm{P}_{19}$ est le plus pollué et nous avons noté une corrélation positive et significative au seuil de $5 \%$ entre les salmonelles et la matière organique $(r=$ $0,45)$ et la matière en suspension $(r=0,50)$. Ceci pourrait se justifier par la proximité du restaurant. En effet, les eaux usées issues des activités du restaurant sont rejetées aux alentours du puits et contaminent l'eau de ce puits par infiltration et lors du puisage. De plus, le puits est ouvert, ce qui favorise la chute des débris végétaux et animaux à l'intérieur, entraînant une élévation de la teneur en matières organiques.

Le nombre de kystes ou d'œufs est inversement proportionnel à la distance entre les puits et les toilettes. En effet, une corrélation négative et significative $(\mathrm{r}=$ $0,66 ; \mathrm{P}=0,002)$ au seuil de $5 \%$ a été déterminée entre le nombre d'œufs d'Ascaris lombricoüdes et la distance entre les toilettes et les puits d'eau. Tel est aussi le cas des œufs de Cryptosporidium $(\mathrm{r}=-0,52 ; \mathrm{p}=0,02)$. Ceci signifierait que les œufs diffuseraient à travers l'eau des installations septiques vers les points d'eau. En plus, les kystes et les oeufs sont rejetés dans l'environnement par les Mammifères dont l'homme, les chats, les chiens, les porcs et les moutons (Bridgman et al., 1995 ; Thompson, 1998 ; El Kettani et Azzouzi, 2006) où ils s'avèrent très résistants et se retrouvent dans les puits par infiltration des eaux de ruissellement. De même, la profondeur des puits influence la densité de la population microbienne. Nous avons trouvé une corrélation négative et significative $(\mathrm{r}=$ $0,51 ; \mathrm{p}=0,02)$ entre les kystes de Giardia lamblia et la profondeur des puits et cela se justifierait par la contamination de l'eau des puits à la suite d'infiltration des eaux de ruissellement.

\section{Conclusion}

Les eaux de puits de la localité de Dang sont acides et très peu minéralisées. Elles sont faiblement polluées par les nitrates et les sulfates alors que le fer, les matières organiques, l'azote ammoniacal et les matières en suspension constituent les principaux facteurs indicateurs de pollution. Les résultats obtenus montrent que les eaux de puits investigués sont chimiquement et microbiologiquement polluées. Ces eaux hébergent de fortes densités de bactéries fécales et de bactéries pathogènes opportunistes et spécifiques d'une part, et des germes parasites d'autre part. Les densités de ces germes subissent d'importantes fluctuations spatiales. Cette étude permet, de ce point de vue, de préciser l'importance de la 
pollution et ses origines potentielles. Les nappes superficielles, accessibles par les puits, apparaissent fortement contaminées par des matières organiques d'origine humaines et animales. Cette pollution trouve très probablement ses origines dans l'insuffisance des infrastructures d'assainissement et de collecte des ordures ménagères. Il est important de mettre en place des structures d'assainissement de l'environnement, mais aussi, il faut mettre un accent particulier sur le comportement, c'est-à-dire l'assimilation et le respect des règles d'hygiène. Dans ce sens, un programme d'information, d'éducation et de communication en direction de la population pour lui faire acquérir des comportements favorables à la préservation, à la potabilité de l'eau depuis la source d'approvisionnement jusqu'à la consommation de l'eau devient une priorité.

\section{REFERENCES}

Aissi MJ. 1992. Impacts des Déchets Domestiques sur la Qualité de la Nappe Phréatique à Cotonou. CPU: Paris ; 69p.

Amegee KP. 1992. Analyse du secteur eau et assainissement au Bénin. - Cotonou : Ministère de la Santé. 93 p.

Angulo FJ, Tippen S, Sharp DJ, Payne BJ, Collier C, Hill JE, Barret TJ, Clark RM, Geldreich EE, Donnel HD, Swerdlow DL. 1997. A community waterborne outbreak of salmonellosis and effectiveness of a boil water order. American Journal of Public Health, 87(4): 580-584.

Balbus JM, Embrey MA. 2002. Risk factors for waterborne enteric infections. Current Opinion in Gastroenterology, 18 (1): 4650.

Bitton G. 1999. Wastewater Microbiology. John \& Sons ; 578p.

Bridgman SA, Robertson RMP, Syed Q, Speed N, Andrews N, Hunter PR. 1995. Outbreak of cryptosporidiosis associated with a desinfected groundwater supply. Epidemiology and Infection. 115(3): 555566.

Cardot C. 2002. Les traitements de l'Eau: Procédés Physico-chimiques et Biologi- ques. Cours et Problèmes Résolus. Ellipses : Paris; 247p.

Cheesbrough M. 1987. Medical Laboratory Manuel for Tropical Countries (Volume I;. second edition). University Press: Cambridge; $605 \mathrm{p}$.

Clausen EM, Gren BL, Litsky W. 1977. Fecal streptococci: indicator of pollution. American Society for Testing and Materials, 635: 247-264.

Close ME, Hodgson LR, Todgre G. 1989. Field evaluation of fluorescent whitening agents and sodium tripolyphosphate as indicator of septic tank contamination in domestic wells. New Zeal. J. Marine Fresh. Res., 23: 563-568.

El Kettani, Azzouzi EM. 2006. Prévalence des helminthes au sein d'une population rurale utilisant les eaux usées à des fins agricoles à Settat (Maroc). Environnement Risques et Santé, 2 : 99106.

Faillat JP. 1990. Origine des nitrates dans les nappes des fissures de la zone tropicale humide : exemple de la Côte d'Ivoire. $J$. Hydrol., 113 : 231-264.

Farrow JAE. 1984. Taxonomic studies of $S$. bovis and $S$. equines. Systematic and Applies Microbiology, 5: 467-482.

Fezeu WML. 2000. Caractérisation physicochimique des sources de l'Adamaoua: cas de quelques «lahorés» de Ngaoundéré et de Tignère. Mémoire de D.E.A. Université de Ngaoundéré. Cameroun. 88 p.

Ford LA. 1994. Detection of Aeromonas salmonicida from water using a filtration method. Aquaculture, 123: 1-7.

Gounot AM. 1994. Microbial Ecology of Groundwater. Academic Press Inc ; 189 219.

Guiraud J, Galzy P. 1980. L'Analyse Microbiologique dans les Industries Alimentaires. Les Editions de l'Usine Nouvelle : Paris ; 157-158.

Hassoune EM, Bouzidi A, Koulali Y, Hadarbach D. 2006. Effets des rejets liquides domestiques et industriels sur la qualité des eaux souterraines au nord de la ville de Settat (Maroc). Bulletin de 
l'Institut Scientifique, Rabat, section Sciences de la Vie, 28: 61-71.

Hassoune EM, El Kettani S, Koulali Y, Bouzidi A. 2010. Contamination bactériologique des eaux souterraines par les eaux usées de la ville de Settat, Maroc. Rev. Microbiol. Ind. San. Environn., 1(4): 1-21.

Havelaar AH, During M, Wersteegh JFM. 1987. Ampicillin-dextrin agar medium for the enumeration of Aeromonas species in water by membrane filtration. J. Appl. Bact., 62: 279-287.

Jayamohan S, Ohgaki S, Hanaki K. 1998. Effect of DO on kinetics of nitrification. Water Supply, 6: 141-150.

Kabler PW, Clark HF. 1961. Coliform group and organisms as indicators of pollution in drinking water. J. Amer. Water Work Association, 53: 1577-1579.

Laferriere M, Minville JJ, Lavoie J, Payment P. 1996. L'industrie porcine et les risques reliés à la santé humaine. Bull. Information Santé Environnem., Québec, 7(2): 1-4.

Le Minor L, Veron M. 1989. Bactériologie Médicale. Flammarion édn: Paris ; $1107 \mathrm{p}$.

Lewa 2005. Evaluation de la qualité des eaux de boisson et de ménage dans le département de la Vina. Mémoire de diplôme d'études approfondies. Université de Yaoundé. 59 p.

Mahendrappa MK. 1989. Impact of forest on water chemistry. Water, Air and Soil Pollution, 46: 61-72.

Membre JM, Burlot PM. 1994. Effects of temperature, $\mathrm{pH}$ and $\mathrm{NaCI}$ on growth and pectinolytic activity of Pseudomonas marginalis. Appl. Environ. Microbiol., 60: 2017-2022.

Mintz E, Reiff F, Tauxe R. 1995. Safe water treatment and storage in the home: a pratical new strategy to prevent waterborne disease. JAMA, 273: 948-953.
Nkhuwa DCW. 2003. Human activities and threats of chronics epidemics in a fragile geologic environment. Physics and Chemistry of the Earth, 28 : 1139-1145.

Nola M, Njine T, Djuikom E, Sikati FV. 2002. Faecal coliforms and faecal streptococci community in the underground water in an equatorial area in Cameroon (Central Africa): the importance of some environmental chemical factors. Wat. Res., 36: 3289-3297.

OMS. 1986. Directives de Qualité pour l'Eau de Boisson. Critères d'Hygiène et Documentation à l'Appui (Vol. 2, $1^{\text {ière }}$ édn). OMS : Genève ; $341 \mathrm{p}$.

OMS. 2000. Directives de Qualité pour l'Eau de Boisson. Critères d'Hygiène et Documentation à l'Appui (Vol. 2, $2^{\mathrm{e}}$ édn). OMS : Genève ; $1050 \mathrm{p}$.

OMS. 2002. Emerging and epidemic-prone diseases. In Global Defence Against the Infectious Disease Threat. World Health Organisation: Geneva; 205 p.

Rodier J. 1978. L'analyse de l'Eau: Eaux Naturelles, Eaux Résiduaires, Eaux de la Mer. Chimie, Physico-chimie, Bactériologie, Biologie (Sixième édition, nouveau tirage). Dunod : Paris ; 1135 p.

SNEC. 2004. Rapport d'activité de la Société Nationale des Eaux du Cameroun (SNEC), Agence de Ngaoundéré. $73 \mathrm{p}$.

Tchotsoua M, Esoh E, Mohamadou G, Ngana JP. 1998. Diagnostic de l'état de l'environnement de Ngaoundéré et contribution pour une approche de gestion. Annales de la Faculté des Arts, Lettres et Sciences Humaines de l'Université de Ngaoundéré : 99-144.

Tchotsoua M, Ndame JP, Wakponou A, Bonvallot J. 1999. Maîtrise et gestion des eaux à Ngaoundéré (Cameroun). Problèmes et esquisses de solutions. GeoEco-Trop., 23 : 91-103.

Thompson RCA. 1998. Giardia Infection. Oxford University Press; 545-561. 